\title{
RPARP-ASI/miR I25a-5p Axis Promotes Cell Proliferation, Migration and Invasion in Colon Cancer
}

\author{
Yongjun Ren $\mathbb{D}^{1, *}$ \\ Caixia Zhao ${ }^{2, *}$ \\ $\mathrm{Yi} \mathrm{He}^{3}$ \\ Xuli Min' \\ Hao Xu' \\ Xiao $\mathrm{Hu}^{\prime}$
}

'Department of Interventional Radiology, Sichuan Key Laboratory of Medical Imaging, Affiliated Hospital of North Sichuan Medical College, Nanchong, Sichuan, 637000, People's Republic of China; ${ }^{2}$ Department of Oncology,

Nanchong Central Hospital, Nanchong, Sichuan, 637000, People's Republic of

China; ${ }^{3}$ Department of Gastrointestinal Surgery, Affiliated Hospital of North Sichuan Medical College, Nanchong, Sichuan, 637000, People's Republic of China

*These authors contributed equally to this work
Background: It was reported that long-noncoding RNAs (lncRNAs) had been identified as a novel class of regulators related to various cancers. RPARP-AS1, a differentially-expressed gene, was found in analysis of the gene expression profile of CRC from GEO database. However, its function has not been clear.

Methods: RPARP-AS1 expression was determined by qPCR and Startbase 3 analysis. Knockdown of RPARP-AS1 in CRC cell lines was performed by RNAi technology, named si-RPARP-AS1 HCT116 and si-RPARP-AS1 LoVo. Cell proliferation was examined by CCK8 and colony formation assay. RNA pull-down and Luciferase reporter assay were performed to confirm the interaction between RPARP-AS1 and miR-125a-5p.

Results: In the study, we found that the expression of RPARP-AS1 was significantly upregulated in CRC tissues and multiple CRC cell lines, which was closely related to poor prognosis of CRC patients. Loss-of-function studies indicated that knockdown of RPARP-AS1 inhibited CRC cell proliferation, migration and invasion in HCT116 and LoVo cell lines. Results of research on the mechanisms showed that RPARP-AS1 functioned as a competitive endogenous RNA (ceRNA) to sponge miR-125a-5p, therefore promoting CRC procession.

Conclusion: In summary, these results indicated that RPARP-AS1/miR-125a-5p axis played a positive role in promoting cell proliferation, migration and invasion in $\mathrm{CC}$. It may be as a biomarker used to evaluate CRC prognosis.

Keywords: RPARP-AS1, miR-125a-5p, colon cancer, lncRNA, proliferation, migration, invasion

\section{Introduction}

Colon cancer is the fourth most commonly diagnosed cancer worldwide, with more than 600,000 deaths estimated each year. ${ }^{1}$ The incidence rate of CRC in city area is far higher than that in rural. ${ }^{2}$ At the time of diagnosis, most of the patients were in advanced stage. ${ }^{3}$ According to the latest statistics in 2015 , the incidence rate of CRC in China accounted for fourth of all cancers, and the mortality rate was fifth, which showed an increasing trend. ${ }^{4}$ The incidence rate of CRC accounts for fourth of all cancers in the United States, while the mortality rate is second. From 2008 to 2011 , incidence rate of CRC dropped by at $4 \%$ or even higher. ${ }^{5}$ The treatment of CRC mainly includes surgery, chemotherapy, radiotherapy, targeted therapy and immunotherapy. ${ }^{6}$ Targeted therapy is known for its safety and efficacy, which can improve the survival time and quality of life of patients. ${ }^{6}$ However, due to the existence of KRAS, NRAS, BRAF or PIK3CA mutations resulting in persistent
Correspondence: Yongjun Ren Department of Interventional Radiology, Sichuan Key Laboratory of Medical Imaging, Affiliated Hospital of North Sichuan Medical College, Nanchong, Sichuan, 637000, People's Republic of China

Email yongjunren_1213@sina.com 
activation, some patients cannot benefit from targeted therapy. ${ }^{7}$ Therefore, it is very important to study the underlying molecular mechanism of CRC and develop new targeting sites and drugs.

Long noncoding RNA (IncRNA) transcripts are between $200 \mathrm{nt}$ and $100 \mathrm{~kb}$ in length. ${ }^{8}$ They do not encode or rarely encode proteins, and have become the focus of scientific research in recent years. The analysis of human genome revealed that IncRNA content was huge, accounting for about $4-9 \% .{ }^{9}$ They play various biological roles by regulating transcription, epigenetics and post-transcriptional translation. The role of lncRNA in the development of disease has been gradually revealed, including cancer, diabetes and nervous system disease. For example, IncRNAs, such as TNXA, CTA-134P22.2, CTC-276P9.1, KRT19p3 and H19 were identified in bladder cancer and adjacent tissues. ${ }^{10} \mathrm{H} 19$ could activate $\mathrm{Wnt} / \beta$-Catenin and down-regulate $\mathrm{E}$-cadherin by binding with EZH2, thus promoting the metastasis of bladder cancer cells. ${ }^{11}$ IncRNA plays a more significant role in the prevention, diagnosis and treatment of CC. Related studies have classified lncRNAs in the course of CC, including PVT1, MALAT-1, ncRNA, CCAT1-1 and PRNCR1. IncRNA HNF1A-AS1 was highly expressed in CC, and promoted cell proliferation through miR-34a/SIRT1/TP53 and Wnt signal transduction pathways. In addition, HNF1A-AS1 was associated with lymph node metastasis. ${ }^{12}$

In a variety of tumors, lncRNA, miRNA and functional gene jointly construct a complex regulatory network to regulate the proliferation, migration, invasion, apoptosis and drug response of tumor cells. In the last few decades, a lot of research had proved that lncRNAs were involved in gene expression network by sponging miRNAs, regulating translation, modifying histone and chromatin, and so on. ${ }^{13,14}$ In the study, we found a differentially-expressed gene, RPARP-AS1, in analysis of the gene expression profile of CRC from GEO database which was positively correlated to poor prognosis of CC. RPARP-AS1 could sponge miR-125a-5p to decrease its abundance in cytoplasm which promoted tumor cell proliferation, migration and invasion.

\section{Methods}

\section{Patients and Specimens}

Thirty patients with CRC in the Affiliated Hospital of North Sichuan Medical College were selected to obtain CRC tissues and corresponding paracancerous tissues. All of the patients had not received any preoperative treatment, including chemotherapy, radiotherapy or targeted therapy which signed informed consent in advance. The obtained specimens were frozen in liquid nitrogen immediately and then transferred to a refrigerator at $-80^{\circ} \mathrm{C}$ for further study. The study was performed under a project license (KE-YT -2018-00) by the Ethics Committee of the Affiliated Hospital of North Sichuan Medical College, which accorded with the ethical standards formulated in the Helsinki Declaration.

\section{Quantitative Real-Time PCR (qRT-PCR)}

Total RNA was extracted by using TRIzol reagent (Thermo Fisher Scientific, United States). The RNA was quantified by using NanoDrop ND2000 microspectrophotometer (Thermo Fisher Scientific, United States). Four microgram of total RNA was then reversely transcribed into cDNA according to the protocol of the Reverse Transcription System (Promega, United States). Quantitative RT-PCR analysis was carried out by using the UltraSYBR Mixture kit (CWBIO, China). In this study, the primers used were synthesized by Sangon Biotech (China). The expression levels of relevant genes were determined by the $\Delta \Delta \mathrm{CT}$ method.

\section{Luciferase Reporter Assay}

For analysis of miR-125a-5p regulated by RPAPP-AS1, the reporter plasmid was constructed by fusing RPAPPAS1 cDNA into the 3'UTR region of Luciferase gene. Then the synthesized miR-125a-5p with the reporter plasmid were co-transfected into HCT116 and LoVo cells. The LUC activities were quantified using the Luciferase Assay System (Promega, United States) which represent the regulation of RPAPP-AS1 on miR-125a-5p.

\section{Cell Transfection}

In order to knock down RPARP-AS1 expression, three siRNA targeting RPARP-AS1 were designed, and three Lentivirus vector containing a siRNA separately were synthesized by BioMiao Biological Technology (Beijing, China), as well as the plasmid for RPARP-AS1 to enhance its expression. HCT116 and LoVo cells were transiently transfected with si-RPARP-AS1, si-NC and OE-RPARPAS1 by using lipofectamine 2000 (Invitrogen, Carlsbad, CA, USA) according to the manufacturer's protocol.

\section{CCK8}

One hundred microlitre cell suspension was inoculated in 96-well plate which was subsequently cultured at $37^{\circ} \mathrm{C}$, 
5\% CO2. Add $10 \mu \mathrm{L}$ CCK 8 solution. The plate continued to be cultured for 1-4 h. Cell proliferation analysis of HCT118 and LoVo cells was performed using a Microplate reader (BioTek, United States).

\section{Colony Formation Assay}

The logarithmic cells were suspended and inoculated in dishes containing culture medium. The dishes were cultured at $37^{\circ} \mathrm{C}$ for $2-3$ weeks until clones being observed. The clones were fixed with $4 \%$ paraformaldehyde and subsequently stained with GIMSA solution for 10-30 $\mathrm{min}$. The clone formation rate was evaluated by formula "Clone formation rate $=$ Number of Clones/Number of Cells $\times 100 \%$ ".

\section{Transwell}

Transwell chamber was put into the culture plate. The upper and lower chambers were filled with liquid medium and solid medium separately. The upper and lower layers of culture medium were separated by a polycarbonate membrane. For the study of cell invasion experiment, EMC was added into the lower medium. The cells were suspended in serum-free medium containing BSA reaching to $5 \times 10^{5} / \mathrm{mL}$. Then $200 \mu \mathrm{L}$ of suspension cells were added into the upper chamber and then cultured in an incubator at $37^{\circ} \mathrm{C}, 5 \% \mathrm{CO} 2$ for $12-48 \mathrm{~h}$.

\section{RNA Pull-Down}

Biotin-labeled miR-125a-5p sense and antisense hybridization probes were obtained by transcription in vitro (Roche, Switzerland). The probes were incubated with cytoplasmic extract from HCT118 and LoVo cells to form miRNA-lncRNA compounds which were separated using magnetic beads labeled with avidin. Finally, qRTPCR analysis determined the enrichment of RPAPP-AS1 in the immunoprecipitated RNA.

\section{Statistical Analysis}

GraphPad Prism software was used to perform Statistical analysis and generate column diagram. The statistically significant differences were evaluated by Student's $t$-test ( ${ }^{*} P$-value $<0.05,{ }^{* *} P$-value $<0.01,{ }^{* * *} P$-value $\left.<0.001\right)$.

\section{Result}

\section{RPARP-ASI Expression Increased in Colon Cancer with Poor Prognosis}

A differentially-expressed gene, RPARP-AS1, was found in analysis of the gene expression profile of CRC from
GEO database. RPARP-AS1 expression was significantly increased in CRC tissues than that in the adjacent tissues (Figure 1A). To confirm this conclusion, 30 pairs of CRC and adjacent tissues were collected in which RPARP-AS1 expression was detected by qRT-PCR. As shown in Figure 1B, RPARP-AS1 expression was significantly higher in CRC tissues than that in the adjacent tissues which was consistent with Figure 1A. Further, we analyzed the relationship between RPARP-AS1 expression and CRC using data from TCGA database by GEPIA. The results demonstrated that highly-expressed RPARPAS1 was greatly correlated with poor prognosis of CRC (Figure 1C). It aroused our interest to study the biological function of RPARP-AS1. Among all cell lines, the expression of RPARP-AS1 was highest in HCT116 and LoVo (Figure 1D), which were selected for further study.

\section{RPARP-ASI Gene Silencing Inhibited Cell \\ Proliferation}

The lentivirus vectors silencing RPARP-AS1 gene expression were introduced into HCT116 and LoVo cell lines separately. As a result, RPARP-AS1 expression significantly decreased (Figure 2A), named si-RPARP-AS1 HCT116 and si-RPARP-AS1 LoVo cell lines. In the course of experiment, we designed three siRNA, in which si\#1 and si\#2 effectively knocked down the expression of RPARP-AS1 (Supplementary Figure 1E), the sequences as follows: si\#1, 5'-AAGAAAAGUAAAUAGGCC-3'; si\#2, 5'-UAAGA AAAGUAAAUAGGC-3'. We selected the vector si\#1 for further study of gene function. Considering that RPARP-AS1 was associated with poor prognosis of CRC (Figure 1C), we guessed that it may be involved in promoting tumor cell proliferation, migration or invasion. Therefore, we performed CCK8, colony formation assay and TransWell to confirm the role of RPARP-AS1 in CC. After $24 \mathrm{~h}$ of culture, the proliferation of si-RPARP-AS1 HCT116 and LoVo cells was lower than that of normal control (si-NC) cells by CCK8. With the extension of culture time, gap was becoming greater and greater until culture for $96 \mathrm{~h}$ (Figure 2B). Consistent with the results of CCK8, si-RPARP-AS1 HCT116 and si-RPARP -AS1 LoVo cells formed fewer clones (Figure 2C). In addition, Transwell results showed that the migration of siRPARP-AS1 HCT116 and LoVo cells significantly reduced than that of si-NC groups (Figure 2D), as well as the invasion of si-RPARP-AS1 cells (Figure 2E). In contrast, overexpression of RPARP-AS1 promoted the proliferation and invasion in HCT116 cells ( $\underline{\text { Supplementary Figure 1A-D) }}$. 
A

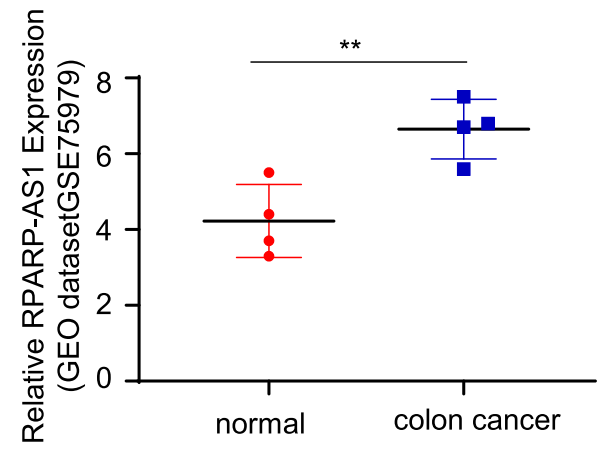

C

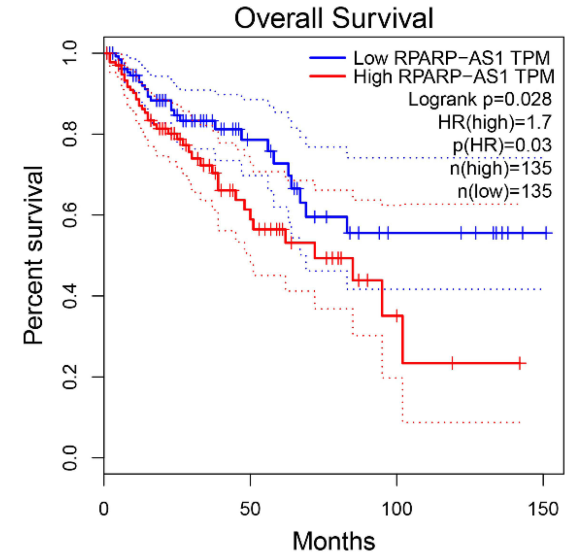

B

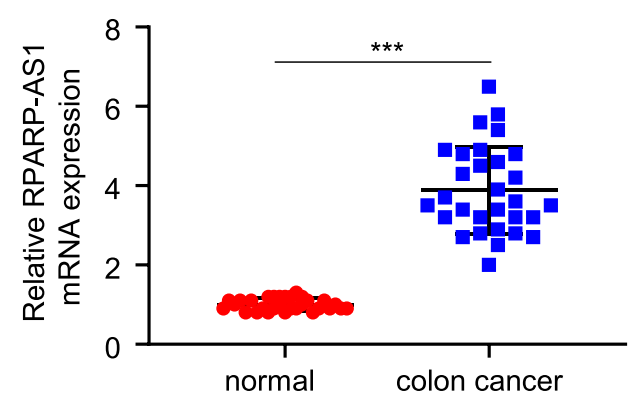

D

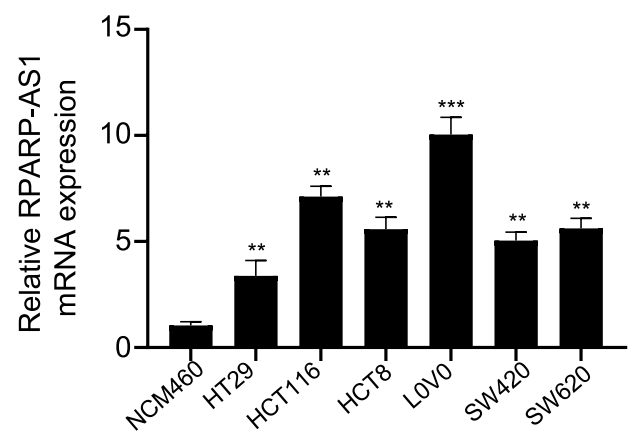

Figure I RPARP-ASI was highly expressed in colon cancer with poor prognosis. (A) RPARP-ASI expression in colon cancer was significantly higher than that in the adjacent tissues from GSE75970 ( $P$-value $<0.01$ ). (B) RPARP-ASI expression in colon cancer than that in the adjacent tissues from clinical samples $(P$-value $<0.00$ I). (C) GEPIA analysis of TCGA database found that patients with high expression of RPARP-ASI had poor prognosis $(P$-value $<0.05)$. (D) RPARP-ASI expression in all colon cancer cell lines (HT29, HCTI 16, hct8, LoVo, sw420, SW620) was higher than that in control group cell line (NCM460) $(* * P$-value $<0.01$; $* * * P$-value $<0.001)$.

\section{RPARP-ASI Sponged miR-125a-5p}

Furthermore, we studied the mechanism of RPARP-AS1 in promoting tumor cell proliferation, migration and invasion in CC. The sub-cellular localization of RPARP-AS1 was explored by Nucleocytoplasmic separation. The results showed that RPARP-AS1 was mainly expressed in cytoplasm (Figure 3A). Combined with previous research, RPARP-AS1 may function by sponging miRNA. The potential miRNA was analyzed by Startbase 3 and the binding site of miR-125a-5p was found in RPARP-AS1 (Figure 3B). RNA pull-down and Luciferase reporter assay were performed to analyze the interaction between RPARP-AS1 and miR-125a-5p. As shown in Figure 3C, miR-125a-5p probe could effectively enrich RPARP-AS1. Also, over-expression of miR-125a-5p could bind to RPARP-AS1 and inhibit the activity of Luciferase in both HCT116 and LoVo cells. Corresponding to it, miR$125 a-5 p$ expression significantly increased in si-RPARPAS1 cells than that in si-NC groups (Figure 3D).
Therefore, RPARP-AS1 sponged miR-125a-5p in CC. In line with expectations, miR-125a-5p expression was lower in CRC using 30 pairs of clinical samples (Figure 3E) and data from TCGA (Figure 3G), which was negatively correlated with RPARP-AS1 expression (Figure 3F and H).

\section{RPARP-ASI Regulated CRC Progression by Sponging miR-125a-5p}

Above results proved that RPARP-AS1 could sponge miR125a-5p directly. Next, we studied the role of RPARP-AS1 /miR-125a-5p axis in CRC procession. As described in Result 2, knockdown of RPARP-AS1 decreased the proliferation, migration and invasion in HCT116 and LoVo cell lines (Figure 2). In this result, miR-125a-5p expression increased in si-RPARP-AS1 cell lines than that in si-NC groups. miR$125 a-5 p$ inhibitor was then introduced in the study which could inhibit miR-125a-5p expression (Figure 4A). In addition, miR$125 \mathrm{a}-5 \mathrm{p}$ inhibitor could reverse decreased tumor cell proliferation by CCK8 (Figure 4B), clone formation by colony 
A

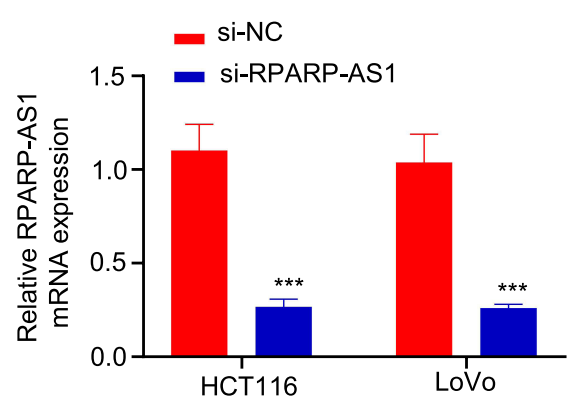

C

HCT116

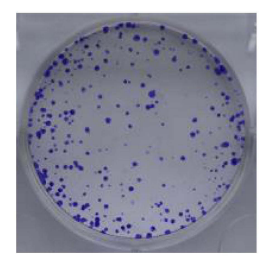

si-NC

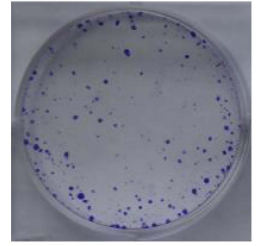

si-RPARP-AS1

D

HCT116

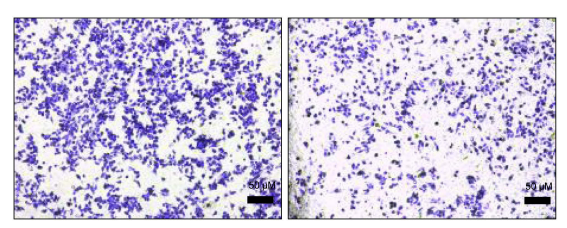

si-NC

si-RPARP-AS1
F

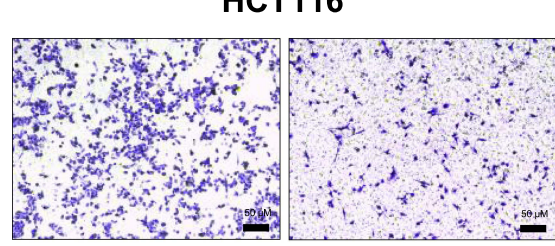

si-NC
si-RPARP-AS1
B
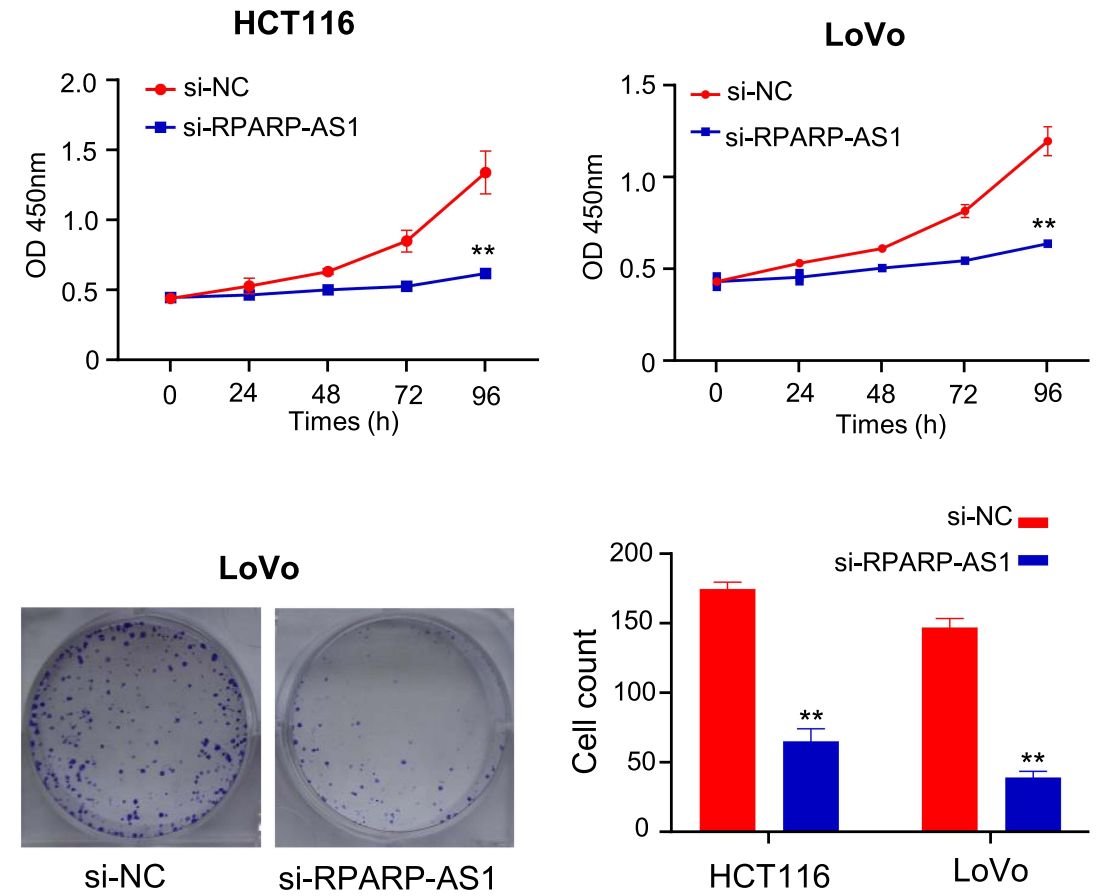

si-NC

\section{LoVo}
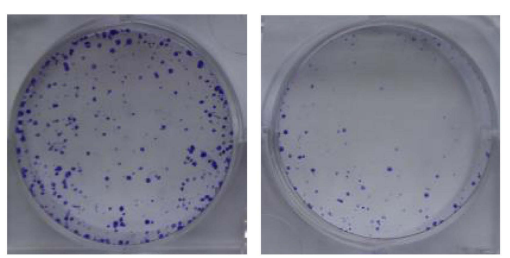

si-RPARP-AS1
HCT116

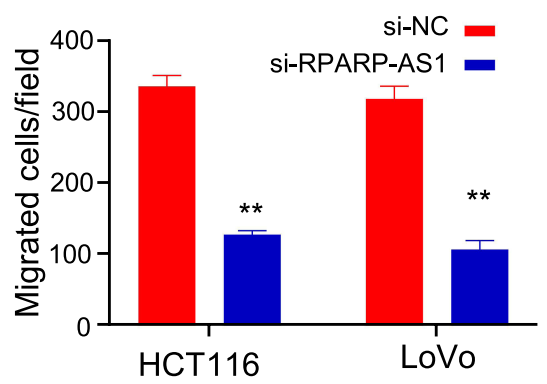

si-NC si-RPARP-AS1

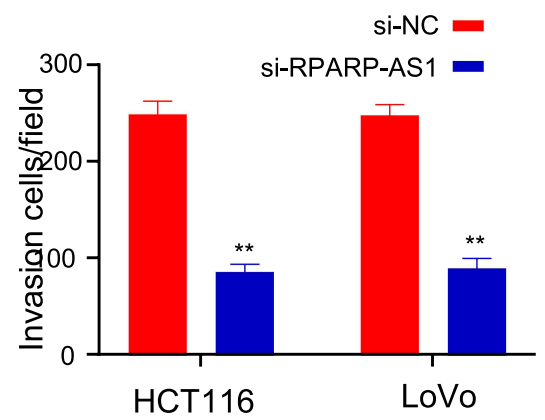

Figure 2 RPARP-ASI knockdown in HCTII6 and LoVo cell lines inhibited cell proliferation. (A) RPARA-ASI expression significantly decreased in both HCTII6 and LoVo cell lines mediated with lentiviral vectors (P-value < 0.00I). (B) CCK-8 was used to detect cell proliferation of HT29 and HCTII6. Knockdown of RPARP-ASI decreased the light absorption value of cells at $450 \mathrm{~nm}$ (P-value $<0.0 \mathrm{I})$. (C) Colony formation assay confirmed that knockdown of RPARP-ASI decreased the colony-forming ability of HCTII 6 and LoVo cells (P-value < 0.0I). (D) TransWell (without Matrigel) showed that knockdown of RPARP-ASI decreased the migration ability of HCTII6 and LoVo cells (P-value $<0.0 \mathrm{I})$. (E) TransWell (with Matrigel) showed that knockdown of RPARP-ASI decreased the invasion ability of HCTII6 and LoVo cells (P-value < $0.0 \mathrm{I})$. (**P-value $<0.01 ; * * *$ P-value $<0.001)$. 
A

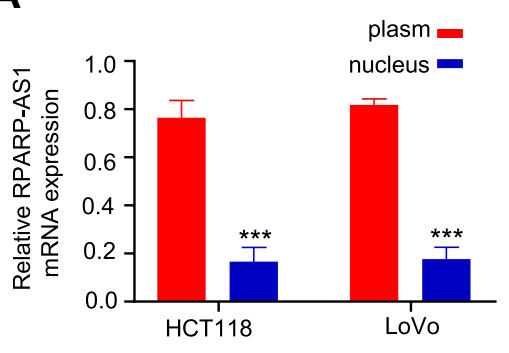

C

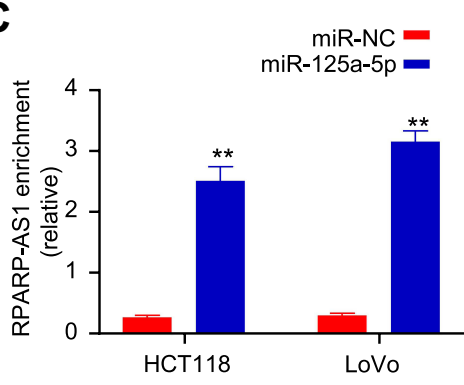

D

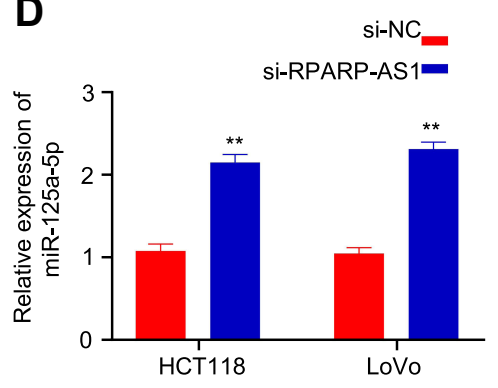

B

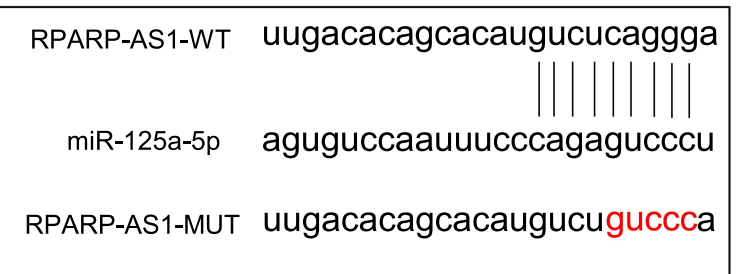

HCT118

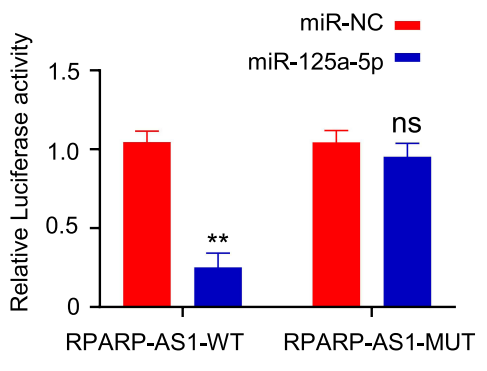

LoVo

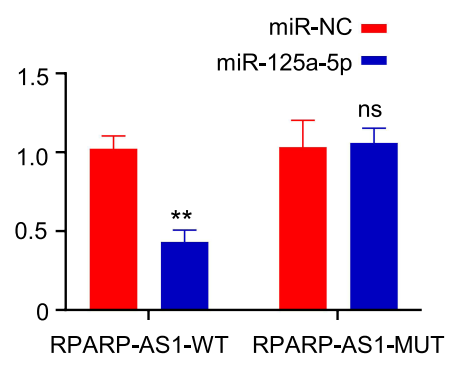

E

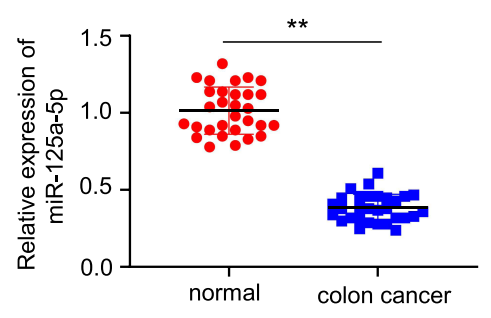

F

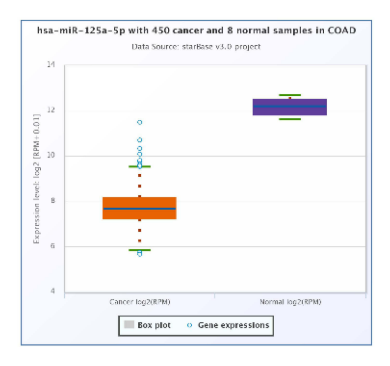

G

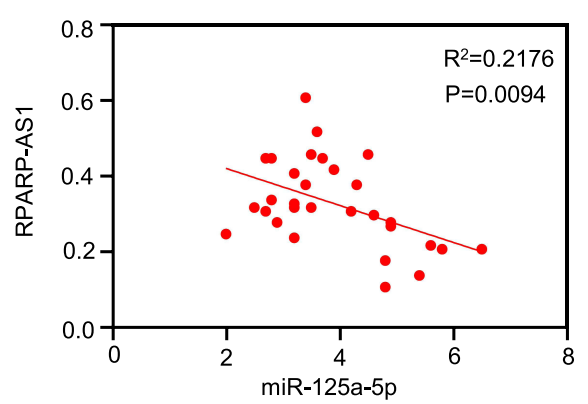

H

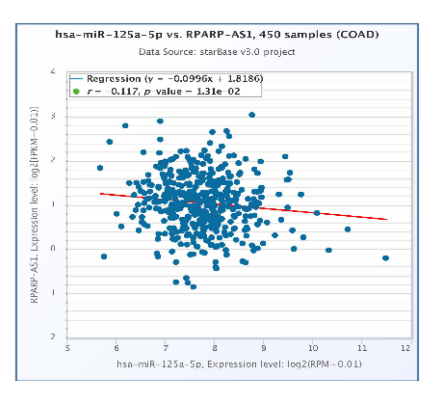

Figure 3 RPARP-ASI sponged miR-125a-5p. (A) RPARP-ASI was mainly expressed in nucleus, but not in cytoplasm (U6 and GAPDH were used as internal parameters of nucleus and cytoplasm respectively). (B) The binding site of miR-125a-5p was found in RPARP-ASI by analyzing the startbase3 database. (C) RNA pull-down assay was performed in HT29 and HCTII6 cells. miR-125a-5p probe enriched more RPARP-ASI than NC probe (Left). Luciferase reporter experiment showed that miR-125a-5p could inhibit the activity of luciferase coupling with RPARP-ASI, but miR-NC could not in both HCTII6 (Middle) and LoVo (Right) cell lines. (D) qRT-PCR was used to detect the expression of mir- $125 \mathrm{a}-5 \mathrm{p}$ after knockdown of RPARP-ASI in HT29 and HCTII6 cells. Compared with si-NC groups, miR-I25a-5p expression increased in siRPARP-ASI cell lines. (E) miR-I25a-5p expression decreased significantly in CRC than that in paracarcinomatic tissue by $q$ RT-PCR ( $n=30$, $P$-value $<0.00 I$ ). (F) Consistent with those in Figure E, miR-125a-5p reduced in CRC significantly using data from TCGA by Startbase3 analysis. (G) The correlation between the expression of RPARP-ASI and miR-I25a-5P in 30 cases of CRC which was negatively correlated ( $P$-value $<0.00 I)$. $(\mathbf{H})$ The expression of RPARP-ASI and miR-I25a-5P in CRC was also negatively correlated using data from TCGA by Startbase3 analysis. (**P-value $<0.01$; ***P-value $<0.00 \mathrm{I}$ ). 

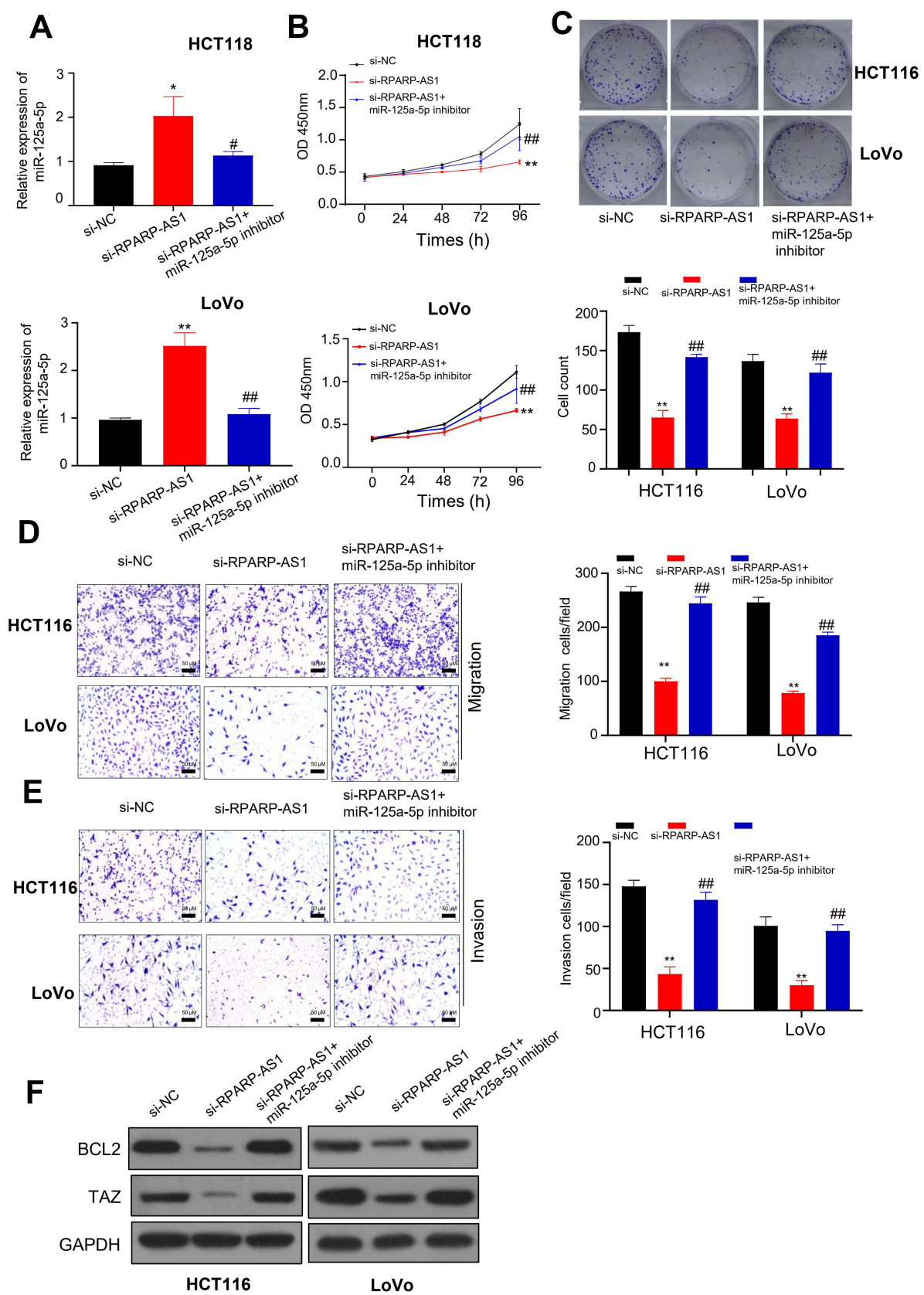

Figure 4 RPARP-ASI sponged miR-I25a-5p to regulate tumor cell proliferation, migration or invasion. (A) miR-I25a-5p expression increased in si-RPARP-ASI cells which partially decreased by miR-125a-5p inhibitor in HCTII6 and LoVo cell lines. (B) The proliferation of si-RPARP-ASI cells decreased than that of si-NC groups which partially increased by miR-125a-5p inhibitor in HCTII6 and LoVo cell lines. (C) Colony formation assay showed that siRPAPR-ASI cells formed fewer clones than si-NC cells which partially increased by miR-125a-5p inhibitor in HCTII6 and LoVo cell lines. (D) TransWell (without Matrigel) showed that knockdown of RPARP-ASI decreased the migration ability of HCTII6 and LoVo cells which was partially recovered by miR-I25a-5p inhibitor. (E) TransWell (with Matrigel) showed that knockdown of RPARP-ASI decreased the invasion ability of HCTII6 and LoVo cells which partially increased by miR-125a-5p inhibitor. (F) Western blot showed that knockdown of RPARP-ASI decreased the expression of BCL2 and TAZ which partially increased by miR-125a-5p. ( ${ }^{* P}$-value $<0.05$, **P-value $<0.01$, compared with si-NC; ${ }^{\# P}$-value $<0.05$, ${ }^{\#} \mathrm{P}$-value $<$ 0.01 , compared with si-RPARP-ASI).

formation assay (Figure 4C), migration (Figure 4D) and invasion (Figure 4E) by TransWell. BCL2 and TAZ were targets of miR-125a-5p in CRC. Thus, we detected the expression of BCL2 and TAZ through Western blot. As shown in Figure 4F, silencing RPARP-AS1 inhibits BCL2 and TAZ expression.
However, when co-transfected with miR-125a-5p, BCL2 and TAZ expression is increased. These results indicated that RPARP-AS1 expression increased which promoting tumor cell proliferation, migration and invasion in CRC by sponging miR-125a-5p. 


\section{Discussion}

lncRNAs are a class of RNA molecules with a length of more than 200 nucleotides. $^{8}$ They do not participate in protein coding, but directly regulate various epigenetics in the form of RNA and participate in transcription and posttranscriptional protein coding. In CRC, lncRNA expression is closely related to the occurrence and development of CRC. In 2011, Graham et al ${ }^{15}$ found that CRNDE-h was upregulated in plasma and tissues of CRC patients. Ellis et al ${ }^{16}$ found that CRNDE affected insulin/insulin-like growth factor (IGF) on regulation of glucose metabolism and lactate secretion through PI3K/Akt/mTOR and Raf/MAPK pathways, whose expression was up-regulated in CRC cells. Dai et $\mathrm{al}^{17}$ further confirmed that CRNDE was highly expressed in tissues, serum and cell lines of CRC patients. It was suggested that CRNDE could activate or inhibit Ras/ MAPK and Wnt/ $\beta$-catenin signaling pathways through competitive binding of miRNA, thus regulating the proliferation, invasion and apoptosis of CRC cells. Therefore, CRNDE might be used as one of the markers for early diagnosis of CRC. ${ }^{18}$ In 2010, Tsang et al ${ }^{10,19}$ found that over-expression of miR-675 promoted the growth of CRC cells, indicating that H19 might be related to the occurrence of CRC. Liang et $\mathrm{al}^{20}$ found that $\mathrm{H} 19$ was highly expressed in mesenchymallike carcinoma cells and primary $\mathrm{CRC}$, and the stable expression of H19 could promote EMT and accelerate tumor growth in vivo and in vitro. Yang et $\mathrm{al}^{21}$ found that H19, as a competitive endogenous RNA (ceRNA) of miR-138 and miR-200a, regulated the expression of multiple genes involved in EMT and accelerates the growth of CRC cells. Schwarzenbach $^{22}$ found the effect of H19 on RB1-E2F pathway and $\mathrm{Cdk} 8-\beta$-Catenin signal transduction. It is speculated that H19, which is highly expressed in primary CRC, can be detected in blood and may be a potential biomarker for diagnosis or prognosis of CRC. Therefore, lncRNA plays an important role in the diagnosis, prognosis, progress and treatment of CRC. In this study, we found a differentially expressed gene, RPARP-AS1, by analyzing the CRC gene expression profile. Consistent with the previous results, RPARP-AS1 was highly expressed in CRC (Figure 1).

In the study, we demonstrated that RPARP-AS1 could promote the proliferation, migration and invasion of tumor cells through sponging mir-125a-5p (Figure 3). Previous studies have shown that mir-125a-5p is downregulated and played a negative role in tumorigenesis and development including colorectal cancer. ${ }^{23-26}$ miR-125a-5p could inhibit the growth, invasion and metastasis of gastric cancer, breast cancer, liver cancer, lung cancer and CRC. ${ }^{27}$ Consistent with these reports, we also found downregulation of miR-125a-5p in CC. As a tumor suppressor, over-expression of miR-125a$5 \mathrm{p}$ inhibited proliferation and induces apoptosis in $\mathrm{CC}$ through targeting BCL2, BCL2L12, MCL- ${ }^{28}$ and TAZ, ${ }^{29}$ which are known as tumor suppressors in CC. In our study, silencing RPARP-AS1 could inhibits BCL2 and TAZ expression, which could be reversed by co-transfected with miR-125a-5p inhibitor. Therefore, RPARP-AS1/miR-125a-5p may be involved in promoting $\mathrm{CRC}$ by regulating the expression of BCL2, BCL2L12, MCL-1 and TAZ genes.

In conclusion, our results demonstrate that RPARPAS1 is highly expressed in $\mathrm{CC}$, which can promote the proliferation, migration and invasion of CRC cells through sponging tumor suppressor miR-125a-5p.

\section{Acknowledgments}

Yongjun Ren and Caixia Zhao are co-first authors for this study.

\section{Disclosure}

The authors report no conflicts of interest in this work.

\section{References}

1. Argiles G, Tabernero J, Labianca R, et al. Localised colon cancer: ESMO clinical practice guidelines for diagnosis, treatment and follow-up. Ann Oncol. 2020;31(10):1291-1305. doi:10.1016/j. annonc.2020.06.022

2. Jahanafrooz Z, Mosafer J, Akbari M, et al. Colon cancer therapy by focusing on colon cancer stem cells and their tumor microenvironment. J Cell Physiol. 2020;235(5):4153-4166. doi: $10.1002 /$ jcp. 29337

3. Auclin E, Zaanan A, Vernerey D, et al. Subgroups and prognostication in stage III colon cancer: future perspectives for adjuvant therapy. Ann Oncol. 2017;28(5):958-968. doi:10.1093/annonc/mdx030

4. Chen W, Zheng R, Baade PD, et al. Cancer statistics in China, 2015. CA Cancer J Clin. 2016;66(2):115-132. doi:10.3322/caac.21338

5. Benson AB 3rd, Venook AP, Cederquist L, et al. Colon cancer, version 1.2017, NCCN clinical practice guidelines in oncology. J Natl Compr Canc Netw. 2017;15(3):370-398. doi:10.6004/jnccn.2017.0036

6. Ruan H, Leibowitz BJ, Zhang L, et al. Immunogenic cell death in colon cancer prevention and therapy. Mol Carcinog. 2020;59 (7):783-793. doi: $10.1002 / \mathrm{mc} .23183$

7. Hsu H-C, Thiam TK, Lu Y-J, et al. Mutations of KRAS/NRAS/BRAF predict cetuximab resistance in metastatic colorectal cancer patients. Oncotarget. 2016;7(16):22257-22270. doi:10.18632/oncotarget.8076

8. Wang X, Yu B, Jin Q, et al. Regulation of laryngeal squamous cell cancer progression by the lncRNA RP11-159K7.2/miR-206/DNMT3A axis. J Cell Mol Med. 2020;24(12):6781-6795. doi:10.1111/ jcmm. 15331

9. Ranzani V, Rossetti G, Panzeri I, et al. The long intergenic noncoding RNA landscape of human lymphocytes highlights the regulation of $\mathrm{T}$ cell differentiation by linc-MAF-4. Nat Immunol. 2015;16 (3):318-325. doi:10.1038/ni.3093 
10. Zhu Y-P, Bian X-J, Ye D-W, et al. Long noncoding RNA expression signatures of bladder cancer revealed by microarray. Oncol Lett. 2014;7(4):1197-1202. doi:10.3892/ol.2014.1843

11. Luo M, Li Z, Wang W, et al. Long non-coding RNA H19 increases bladder cancer metastasis by associating with EZH2 and inhibiting E-cadherin expression. Cancer Lett. 2013;333(2):213-221. doi:10.1016/j.canlet.2013.01.033

12. Fang C, Qiu S, Sun F, et al. Long non-coding RNA HNF1A-AS1 mediated repression of miR-34a/SIRT1/p53 feedback loop promotes the metastatic progression of colon cancer by functioning as a competing endogenous RNA. Cancer Lett. 2017;410:50-62. doi:10.1016/j.canlet.2017.09.012

13. Ponjavic J, Ponting CP, Lunter G. Functionality or transcriptional noise? Evidence for selection within long noncoding RNAs. Genome Res. 2007;17(5):556-565. doi:10.1101/gr.6036807

14. Geisler S, Coller J. RNA in unexpected places: long non-coding RNA functions in diverse cellular contexts. Nat Rev Mol Cell Biol. 2013;14 (11):699-712. doi:10.1038/nrm3679

15. Graham LD, Pedersen SK, Brown GS, et al. Colorectal Neoplasia Differentially Expressed (CRNDE), a novel gene with elevated expression in colorectal adenomas and adenocarcinomas. Genes Cancer. 2011;2(8):829-840. doi:10.1177/1947601911431081

16. Ellis BC, Graham LD, Molloy PL. CRNDE, a long non-coding RNA responsive to insulin/IGF signaling, regulates genes involved in central metabolism. Biochim Biophys Acta. 2014;1843(2):50-62. doi:10.1016/j.bbamcr.2013.10.016

17. Dai M, Li S, Qin X. Colorectal neoplasia differentially expressed: a long noncoding RNA with an imperative role in cancer. Onco Targets Ther. 2018;11:3755-3763. doi:10.2147/OTT.S162754

18. Hongzhen Z, Yanyu L, Xuexiang L, et al. The diagnostic and prognostic significance of long non-coding RNA CRNDE in pan-cancer based on TCGA, GEO and comprehensive meta-analysis. Pathol Res Pract. 2019;215(2):256-264. doi:10.1016/j.prp.2018.11.008

19. Tsang WP, Ng EKO, Ng SSM, et al. Oncofetal H19-derived miR-675 regulates tumor suppressor $\mathrm{RB}$ in human colorectal cancer Carcinogenesis. 2010;31(3):350-358. doi:10.1093/carcin/bgp181

20. Liang W-C, Fu W-M, Wong C-W, et al. The lncRNA H19 promotes epithelial to mesenchymal transition by functioning as miRNA sponges in colorectal cancer. Oncotarget. 2015;6(26):22513-22525. doi:10.18632/oncotarget.4154
21. Yang W, Ning N, Jin X. The lncRNA H19 promotes cell proliferation by competitively binding to miR-200a and derepressing beta-catenin expression in colorectal cancer. Biomed Res Int. 2017;2017:2767484

22. Schwarzenbach H. Biological and clinical relevance of H19 in colorectal cancer patients. EBioMedicine. 2016;13:9-10. doi:10.1016/j. ebiom.2016.11.001

23. Liu H, Ma Y, Liu C, et al. Reduced miR-125a-5p level in non-smallcell lung cancer is associated with tumour progression. Open Biol. 2018;8(10):180118. doi:10.1098/rsob.180118

24. Cai M, Chen Q, Shen J, et al. Retracted: epigenetic silenced miR-125a-5p could be self-activated through targeting Suv39H1 in gastric cancer. J Cell Mol Med. 2018;22(10):4721-4731. doi:10.1111/ jcmm. 13716

25. Tang L, Zhou L, Wu S, et al. MiR-125a-5p inhibits colorectal cancer cell epithelial-mesenchymal transition, invasion and migration by targeting TAZ. Onco Targets Ther. 2019;12:3481-3489. doi:10.2147/OTT.S191247

26. Wang L-B, Feng L, He J, et al. MiR-125a-5p inhibits the proliferation and invasion of breast cancer cells and induces apoptosis by targeting GAB2. Math Biosci Eng. 2019;16(6):6923-6933. doi:10.3934/ mbe. 2019347

27. Naidu S, Shi L, Magee P, et al. PDGFR-modulated miR-23b cluster and miR-125a-5p suppress lung tumorigenesis by targeting multiple components of KRAS and NF-kB pathways. Sci Rep. 2017;7 (1):15441. doi:10.1038/s41598-017-14843-6

28. Tong Z, Liu N, Lin L, et al. MiR-125a-5p inhibits cell proliferation and induces apoptosis in colon cancer via targeting BCL2, BCL2L12 and MCL1. Biomed Pharmacother. 2015;75:129-136. doi:10.1016/j. biopha.2015.07.036

29. Yang M, Tang X, Wang Z, et al. miR-125 inhibits colorectal cancer proliferation and invasion by targeting TAZ. Biosci Rep. 2019;39 (12):BSR20190193.
OncoTargets and Therapy

\section{Publish your work in this journal}

OncoTargets and Therapy is an international, peer-reviewed, open access journal focusing on the pathological basis of all cancers, potential targets for therapy and treatment protocols employed to improve the management of cancer patients. The journal also focuses on the impact of management programs and new therapeutic
Dovepress

agents and protocols on patient perspectives such as quality of life, adherence and satisfaction. The manuscript management system is completely online and includes a very quick and fair peer-review system, which is all easy to use. Visit http://www.dovepress.com/ testimonials.php to read real quotes from published authors. 